\title{
High throughput in vitro seed germination screen identified new $A B A$ responsive RING-type ubiquitin E3 ligases in Arabidopsis thaliana
}

\author{
M. Pavicic ${ }^{1,2}$ (D) F. Wang ${ }^{1}$ (D) K. Mouhu ${ }^{1,2}$ (D) K. Himanen ${ }^{1,2}$ (D)
}

Received: 16 October 2018 / Accepted: 16 September 2019 / Published online: 20 September 2019

(c) The Author(s) 2019

\begin{abstract}
Seed quality is an important factor for seedling vigour as well as adult plant resilience. The key quality attributes are related to physical characteristics, physiological performance, genetic background and health status of the seeds. Many ways to address seed quality attributes have been developed and recently many of them have featured automated high throughput methods. In our study, we addressed two of the seed quality attributes, namely physiological performance and genetic background by analysing germination rates in our mutant collection. These mutants represent ubiquitin E3 ligases that transcriptionally respond to abscisic acid (ABA). This plant hormone is an important regulator of germination and seedling establishment. To facilitate in vitro germination screens of large seed collections a high throughput image-based assay was developed. As a read out of the germination on ABA treatment the cotyledon emergence was detected with top view chlorophyll fluorescence camera. By applying the ABA treatment during germination, RING-type ubiquitin E3 ligase mutants were identified, showing either resistant or sensitive responses to ABA. In conclusion, a scalable high throughput screen for in vitro germination assay was established that allowed fast screening of tens of mutants in a hormone supplemented media.
\end{abstract}

\section{Key message}

To assign ubiquitin E3 ligases to ABA pathway a high throughput chlorophyll fluorescence-based germination assay was established. Screen identified novel ABA response ubiquitin E3 ligases with opposite responses.

Keywords Seed germination · ABA · Plant phenotyping · Chlorophyll fluorescence · Arabidopsis · In vitro · RING-type ubiquitin E3 ligases

Communicated by Ming-Tsair Chan.

Electronic supplementary material The online version of this article (https://doi.org/10.1007/s11240-019-01700-9) contains supplementary material, which is available to authorized users.

K. Himanen

kristiina.himanen@helsinki.fi

M. Pavicic

Mirko.pavicic@helsinki.fi

F. Wang

Feng.z.wang@helsinki.fi

K. Mouhu

katriina.mouhu@helsinki.fi

1 Department of Agricultural Sciences, Faculty of Agricultural Sciences, University of Helsinki, Latokartanonkaari 7 , 00014 Helsinki, Finland

2 National Plant Phenotyping Infrastructure, Faculty of Biological and Environmental Sciences, University of Helsinki, Helsinki, Finland

\section{Introduction}

Plants are sessile organisms that have developed a wide array of response pathways to meet the challenges imposed by the environment. Characterization of these response pathways holds a key to genetic resources that can be utilized in molecular breeding. Among all signalling pathways, the ubiquitin proteasome system (UPS) mediated degradation of selected substrates has emerged as a powerful regulatory mechanism for switching between growth and stress responses (Sadanandom et al. 2012). The UPS system is based on tagging substrate protein with a chain of ubiquitin proteins to act as a signal for $26 \mathrm{~S}$ proteasome mediated degradation. For the attachment of ubiquitin, a cascade of three enzymatic reactions are required, by ubiquitin activating enzyme (E1), ubiquitin conjugating enzyme (E2), and ubiquitin ligase enzyme (E3). Genetic analyses in plants have proposed that the UPS plays a vital role in numerous of 
signalling pathways; including floral homeostasis (Ni et al. 2004), pathogen defence (Gou et al. 2017), stress responses (Tian et al. 2015; Adler et al. 2017), and phytohormone signalling (Gray et al. 1999; Tan et al. 2007). Apart from the hormone receptors, the majority of the UPS signalling pathway networks still remain to be elucidated.

The E3 ligases are the most abundant UPS components; there are more than a thousand E3 ligase genes in Arabidopsis genome (Stone et al. 2005; Jiménez-López et al. 2018). The E3 ligases found in plants belong to one of seven subtypes that can be divided in two: genes with either a Homology to E6-AP C-Terminus (HECT) or Really Interesting New Gene (RING)/U-box domain (Sadanandom et al. 2012; Jiménez-López et al. 2018). The RING-type E3 proteins are the most abundant among the single subunit E3 ligases and can be divided in seven RING finger domain classes distributed in 30 groups (Stone et al. 2005; Jiménez-López et al. 2018). However, only few E3 ligases have been functionally analysed and their substrates identified.

Spatio-temporal gene expression patterns are informative in assigning proteins to their signalling and regulatory pathways. Recently, we have curated the Arabidopsis RING-type ubiquitin E3 ligase protein sequence number to 509 and associated 60 out of them to floral organ or flowering time regulation based on their expression patterns in the Genevestigator database (Hruz et al. 2008; Pavicic et al. 2017). Through these associations, we identified four novel flowering time or flower development regulators. In the current study, we analysed gene expression patterns of a collection of RING-type ubiquitin E3 ligase genes. Several of the genes were responding to various perturbations and signalling pathways, such as abiotic and biotic stress factors and phytohormones. These results support the hypothesis that ubiquitin E3 ligases play multiple roles in and integrate different signalling pathways (Sadanandom et al. 2012; Stone 2014). To further investigate the role of these genes in hormone pathways, we analysed their expression patterns in abscisic acid (ABA) and gibberellic acid (GA) during germination. To confirm the functional importance of the hormonal responses at gene expression level, we developed a hormone response assay using (ABA) as an early regulator of germination and seedling emergence (Vishal and Kumar 2018).

We utilized the National Plant Phenotyping Infrastructure (NaPPI, https://www.helsinki.fi/en/infrastructures/ national-plant-phenotyping) for developing an experimental design and workflow for image-based high throughput in vitro screen. Various platforms and sensors have been used previously for germination analysis, including RGB, VNIR and SWIR (Joosen et al. 2010; Whan et al. 2014; Dumont et al. 2015; Jahnke et al. 2016; Ugena et al. 2018). Here, we introduce a novel approach that utilizes a conveyor belt-based system to deliver thousands of seeds under an imaging station to be scored in one experimental round. Due to the small size of Arabidopsis seeds a chlorophyll fluorescence-based imaging system was used to detect the emerging cotyledons (principle growth stage 0.7 of seed germination according to Boyes et al. 2001) as indication of successful seed germination on ABA containing media. Through screening the selected mutant collection, we identified several RING-type ubiquitin E3 ligases mutant lines with altered ABA responses compared to wild type Col0 . One mutant showed resistant response to ABA, while other four were susceptible to ABA, suggesting that the E3 ligases can act as both positive and negative regulators of ABA responses during germination and seedling emergence. These observations support the emerging versatile roles for RING-type E3 ligases in regulation of ABA response components, further expanding the current signalling models of ABA responses (Yu et al. 2016). The developed system can be easily extended to screening responses to any hormones and natural or chemical compounds or treatments.

\section{Materials and methods}

\section{Bioinformatic analysis}

Genevestigator gene expression database software was used to associate RING-type ubiquitin E3 ligases with ABA signalling (https://genevestigator.com/gv/) by selecting a set of microarray experiments where Arabidopsis seedlings or organs were treated with ABA (AT-00110, AT-00218, AT-00231, AT-00241, AT-00420, AT-00433, AT-00468, AT-00539, AT-00541 and AT-00637). Expression data from these experiments were retrieved and sorted for 36 RINGtype ubiquitin E3 ligases, and 15 of these with more than fourfold of change $\left(\mathrm{LOG}_{2}>2\right)$ where selected for further analysis. For the fifteen ABA responding RING-type ubiquitin E3 ligase genes, gene expression data of a time course analysis of Arabidopsis seed treatment with gibberellic acid (GA), was extracted from GSE5701 dataset (Goda et al. 2008) in the Gene Expression Omnibus (GEO) database.

\section{Plant material and hormone treatments}

In total, 18 Arabidopsis Col-0 mutant lines representing the 15 ABA responding genes were obtained from the NASC stock centre. Genotyping of the mutant lines (Table 1) was performed as described in Pavicic et al. (2017). For germination assays, seed stocks were harvested from three individual plants per line grown together in a growth chamber set at $16 \mathrm{~h} \mathrm{light} / 8 \mathrm{~h}$ darkness, $22{ }^{\circ} \mathrm{C}$, and $60 \%$ relative air humidity. These three seed stocks per line were considered as biological replicates. In vitro plants were grown on $1 / 2 \mathrm{MS}$ (Duchefa Biochemie, The Netherlands, www.duchefa-bioch 
Table 1 List of genes and mutant lines analysed in this study

\begin{tabular}{llllll}
\hline Locus & Common name & Mutant name & NASC accession & Mutant code & Mutant status \\
\hline AT1G05880 & ARI12 & ari12-1 & N685593 & SALK_033142C & KO \\
AT1G63840 & - & at1g63840-1 & N344073* & GK-299B05.01 & KO \\
AT1G68820 & PPRT1 & pprt1-2 & N667194* & SALK_122743C & KO \\
AT1G76410 & ATL8 & atl8-1 & N530639* & SALK_030639 & KO \\
AT1G77770 & - & at1g77770-1 & N2007587 & GK-950G02.02 & NC \\
AT2G22680 & WAVH1 & wavh1-1 & N653622* & SALK_041291C & KO \\
& & wavh1-2 & N219965 & CATMA2a21170 & Down \\
AT3G01650 & RGLG1 & rglg1-1 & N655271* & SALK_011892C & KO \\
AT3G46620 & RDUF1 & rduf1-1 & N663927 & SALK_129323C & NC \\
AT4G11360 & RHA1B & rhalb-1 & N678410 & SALK_042748C & Down \\
& & rhalb-2 & N678848 & SALK_094834C & Down \\
AT4G14365 & XBAT34 & xbat34-1 & N873757 & SAIL_395_E02 & KO \\
AT4G28890 & ATL42 & atl42-1 & N664363 & SALK_152150C & NC \\
& & atl42-2 & N203634* & GK-408A01.01 & KO \\
AT5G01520 & AIRP2 & airp2-3 & N24976* & SALK_053790 & Down \\
AT5G20910 & AIP2 & aip2-1 & N643574 & SALK_143574 & KO \\
AT5G27420 & ATL31/CNI1 & atl31-2 & N339285* & GK-476A11.02 & NC \\
AT5G41400 & - & at5g41400-1 & N306029* & GK-479B03.11 & Down \\
\hline
\end{tabular}

Mutant status refers to the RT-qPCR analysis of the expression of genes with T-DNA insertions

KO knockout, Down downregulated, $N C$ no change

*Mutant lines used in Pavicic et al. (2017) emie.com), $500 \mathrm{mg} / \mathrm{L}$ MES buffer, $10 \mathrm{~g} / \mathrm{L}$ sugar, $0.8 \mathrm{~g} / \mathrm{L}$ agar, pH 5.7 adjusted with $\mathrm{KOH}$. For the ABA screening assay development, media was supplemented with $0 \mathrm{nM}$, $250 \mathrm{nM}, 500 \mathrm{nM}$ or $1000 \mathrm{nM}$ of ABA (Sigma-Aldrich, Germany, www.sigmaaldrich.com) in $10 \mathrm{~cm}$ petri dishes (Sarstedt, Germany, www.sarstedt.com). Fresh ABA stock was used for every experiment. 30 seeds per plate was used to ensure that the emerging cotyledons did not touch or overlap upon germination on the taken images. Seeds were stratified for three nights at $+4{ }^{\circ} \mathrm{C}$ in dark, and transferred to the growth chamber (PhytoScope, PSI, Czech Rep., www.psi. cz) to $16 \mathrm{~h} \mathrm{light} / 8 \mathrm{~h}$ darkness, even $22{ }^{\circ} \mathrm{C}$, and $60 \%$ relative air humidity. The light condition was set and controlled at $130 \mu \mathrm{mol} \mathrm{m} \mathrm{m}^{-2} \mathrm{~s}^{-1}$ photon flux of photosynthetically active radiation (PAR), using a LI-190R Quantum Sensor coupled to LI-250A light meter (LI-COR, Germany, www.licor.com).

For a progressive drought analysis seeds were sown in high density ( 60 seeds per $8 \mathrm{~cm} \times 8 \mathrm{~cm}$ pots) directly on soil with 50\% 1-2 mm vermiculite (Vermipu Oy, Finland) and 50\% peat (Kekkilä kylvöseos W HS R8017, Kekkilä Oy, Finland), and watered until saturation. The pots were placed on trays with plastic domes to keep high humidity and cold stratified at $4{ }^{\circ} \mathrm{C}$ in dark for three nights. After stratification, trays were transferred to growth chamber with $12 \mathrm{~h}$ light/12 h darkness (PhytoScope, PSI, Czech Rep., www. psi.cz). Light intensity, temperature and relative humidity were the same as stated above. Four days after stratification (DAS), tray domes were removed. Seven days after stratification, seedlings were transferred to individual pots watered until saturation and separated into two groups: well-watered and progressive drought. Well-watered group was set to keep watering to 55\% water content based on pot weight, while progressive drought was left to dry until drought symptoms became clear. Imaging trays of 20 plants each ( $7 \mathrm{~cm}$ distance between plants) were lifted few centimetres from the shelves to ensure an even pot drying by allowing air circulation through tray bottom holes. Soil was covered with blue sand (Komodo CaCo Royal Blue Sand, www.Komodoproducts.com) to improve automated plant recognition. Quenching analysis of chlorophyll fluorescence and RGB images were taken to assess drought progression (Abid et al. 2017; Tschiersch et al. 2017). Imaging, image processing and data analysis of these plants were performed as described in Pavicic et al. (2017).

\section{RT-qPCR gene expression}

Two weeks old in vitro grown Col-0 wild type seedlings were collected and incubated in $0 \mu \mathrm{M}$ or $100 \mu \mathrm{M}$ ABA solution in $1 / 2$ MS with gentle agitation for $0,1.5,3$ and $6 \mathrm{~h}$. Three biological replicates were prepared for each ABA concentration and time point. RNA was extracted using InviTrap ${ }^{\circledR}$ Spin Plant RNA Kit (STRATEC Molecular, Germany, www.invitek-molecular.com), cDNA was prepared with SuperScript ${ }^{\circledR}$ IV Reverse Transcriptase (Thermo Fisher Scientific, Inc. www.thermofisher.com), and qPCR 
was performed using Roche Lightcycler ${ }^{\circledR} 480$ Instrument II with LightCycler ${ }^{\circledR} 480$ SYBR Green I Master (Roche Diagnostics, Switzerland. www.roche.com). The fold up values were calculated using the $2^{-\Delta \Delta \mathrm{CT}}$ method according to Schmittgen and Livak (2008), using one of the biological replicates at $0 \mathrm{~h}$ as baseline. Reference genes in this study were two stable genes across many conditions according to Czechowski et al. (2005): TIP41 LIKE (AT4G34270) and AT4G33380. The primers for each gene and the thermal protocols are presented in Supplemental Table S1.

\section{High throughput in vitro germination assays}

The compact phenotyping facility NaPPI at the University of Helsinki Viikki campus greenhouses (https://www.helsi nki.fi/en/infrastructures/national-plant-phenotyping/nappi -facilities) was used to design the high throughput in vitro germination assays. In one experimental round, 30 seeds were sown on a petri dish, one per treatment and biological replication. In the ABA germination assays, in total 5400 seeds representing the 18 mutant lines and Col- 0 wild type, were sown and the plates were fully randomized on the 27 trays, each holding 9 dishes (Fig. 1a). The germinating seeds were imaged daily by an overhead pulse amplitude modulated (PAM) chlorophyll fluorescence camera for 7 days (FluorCam, PSI, Czech Rep., www.psi.cz, Fig. 1a).

\section{Image processing}

Detection of germination was done based on chlorophyll fluorescence emitted from the emerging cotyledons from the germinating seed. The imaging capture was done using a $\mathrm{F}_{\mathrm{v}} / \mathrm{F}_{\mathrm{m}}$ imaging protocol of a FluorCam system (PSI, Czech Rep., www.psi.cz) that generates parameter images of minimum fluorescence $\mathrm{F}_{\mathrm{o}}$ and maximum fluorescence $\mathrm{F}_{\mathrm{m}}$ (Murchie et al. 2013; Tschiersch et al. 2017). This technique is based on dark-adaptation of plants (15 min) to open the photosystem II (PSII) centres. Then, plants are irradiated with a soft measuring light pulse that is not able to trigger photosynthesis, but it is enough to generate a minimum of chlorophyll fluorescence. At this moment an image is taken and saved as $\mathrm{F}_{0}$ (minimal fluorescence). A saturating light pulse generates a maximum peak of chlorophyll fluorescence. Here, a second image is taken and stored as $\mathrm{F}_{\mathrm{m}}$ (maximum fluorescence; Murchie et al. 2013; Tschiersch et al. 2017). $\mathrm{F}_{\mathrm{m}}$ parameter is very effective to detect small cotyledons due to their chlorophyll content. The background fluorescence is almost non-existent and easily removed, making these images suitable for automated object detection and counting (Fig. 1b). To extract pixel information, we processed the $F_{m}$ images with "Fiji is just ImageJ" (FIJI) software (Schindelin et al. 2012; Rueden et al. 2017). The images were imported to FIJI software using "import RAW" command and saved as.tiff images for post processing. The pixel cut off to select only the green pixelated seedlings from the $\mathrm{F}_{\mathrm{m}}$ pictures were determined each time the camera settings were modified to avoid over or under representation. To discard any noise from the pictures, all pixels with values below 180 were filtered out by the function "threshold" (Fig. 1b). Binary images containing two values for each pixel, 0 for the background and 255 for the object-of-interest, were generated (Fig. 1b). On binary images, a rectangle was drawn around each plate in $\mathrm{F}_{\mathrm{m}}$ images and seedlings were counted using FIJI function "particle analysis" (Fig. 1b). The process from thresholding to particle analysis was automated using FIJI macro record function and process folder template from FIJI script editor. Scripting the functions allowed processing of dozens of pictures in couple of minutes, task that would take hours or days when done manually. The output of the image analysis was a CSV file with a column for the image name and another column with the seedling count. All these processes were summarized in three scripts and they are available at GitHub repository (https://github.com/mipavici/ PCTOC-Pavicic-2019).

The image name contained information about the experiment number, measuring round, experiment name, hormone concentration and biological replicate number. The image files were imported to R (https://www.r-project.org/), where the image name was separated in multiple columns for better access to the information and a new column was added giving position names to each measurement from $\mathrm{A} 1$ to $\mathrm{C} 3$. This spreadsheet was bound to another containing mutant lines names by the function "inner_join of dplyr" package using position and tray names as binding references. This process created the final data file that was used for further analyses. Once the process pipeline was assembled, it was possible to go from 500 raw images per experimental round to exploratory plots in few minutes.

\section{Manual validation of the germination screen}

To verify the reliability of the automated cotyledon emergence screening, a manual germination count was performed by sowing seeds on 24 petri dishes containing $1 / 2 \mathrm{MS}$ media without $\mathrm{ABA}$ and 24 plates supplemented with $250 \mathrm{nM}$ ABA. The cotyledon emergence was counted manually for 7 days. Parallel, $\mathrm{F}_{\mathrm{m}}$ pictures of all the petri dishes were recorded daily. $\mathrm{F}_{\mathrm{m}}$ images were used for automatic cotyledon emergence counting as it was described in the previous section. Altogether, germination of a total of 336 individual seeds were counted for manual and automated assays and the percentage of germination was calculated. To establish the relationship between manual and automated counting a plot of manual versus automated percentage of cotyledon emergence was generated and a linear regression model was fitted. 
a

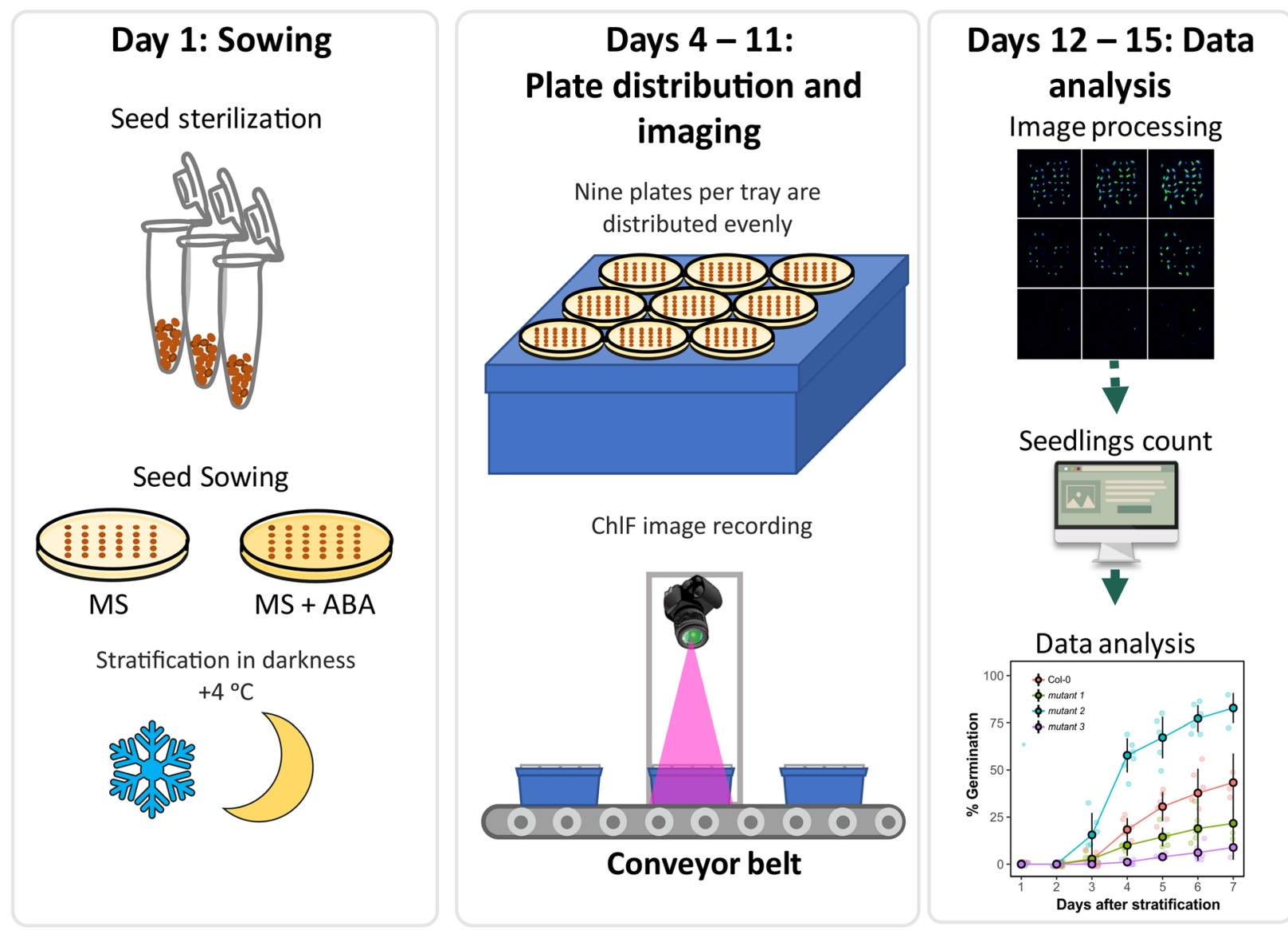

b
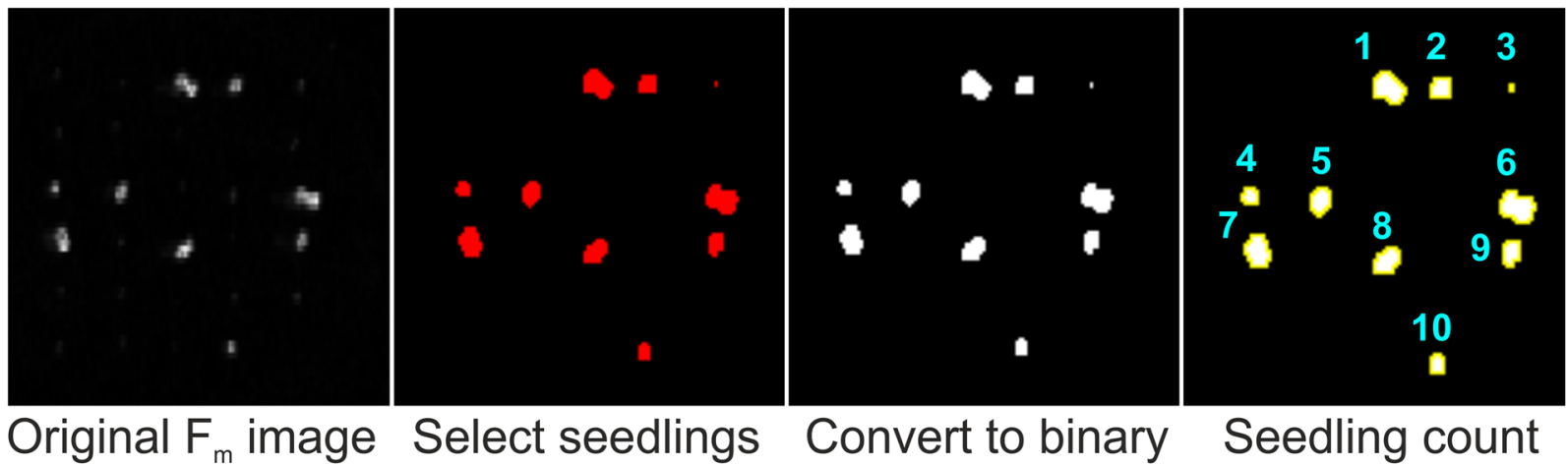

Select seedlings

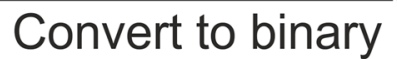

Seedling count

by pixel thresholding
Fig. 1 Work flow of the high throughput in vitro chlorophyll fluorescence imaging system. a Day 1: surface sterilization of the seedsplating on petri dishes with solidified MS or MS supplemented with ABA - stratification of seeds in cold room-in darkness for three nights. Days 4-11: Three petri dishes with 30 seeds per genotype distributed on the PlantScreen trays-daily imaging for chlorophyll fluorescence (ChlF). Days 12-15: collection of .fimg images-image processing for germinated seedling count-time course data analysis to determine $\%$ of germination per genotype. b Image processing steps in FIJI program, from left to right, first: fluorescence max $\left(\mathrm{F}_{\mathrm{m}}\right)$ .fimg image of all germinated Arabidopsis seedlings on a petri dish, second: pixel thresholding based on maximum fluorescence values, third: binary image generated by assigning 0 value to background pixels and 255 to seedling pixels, fourth: automatic seedling count 


\section{Statistical analysis of the data}

Rstudio (Version 1.1.383) Software was used to process and statistically analyse the percentage of germination per treatments, plates and trays (https://www.r-project.org/). Daily differences of percentage of germination and maximum germination differences were tested using ANOVA test with aov function of $\mathrm{R}$ base package. The experiment was repeated two times and the repetition was included in the model as a block. Statistical differences were calculated using Dunnett post hoc test with the function ghlt from multcomp package (Hothorn et al. 2008). Probability values were corrected taking into consideration the number of comparisons.

\section{Results}

\section{Data mining for ABA responsive RING-type ubiquitin E3 ligases}

RING-type Ubiquitin E3 ligases are a large protein family with diverse additional domains determining the upstream regulation and downstream targets (Stone et al. 2005; Jiménez-López et al. 2018). Through the complex structural characteristics RING-type ubiquitin E3 ligases are involved in many developmental or signalling processes, including ABA (Sadanandom et al. 2012; Yu et al. 2016). Here, we searched for ABA signalling components by submitting 36 RING-type ubiquitin E3 ligase genes to ABA related microarrays in Genevestigator (Hruz et al. 2008). As a control we included ABI3 INTERACTING PROTEIN 2 (AIP2), a RINGtype ubiquitin E3 ligase gene negatively regulating ABA signalling during germination (Zhang et al. 2005). Of 36 RINGs, 15 showed responses to ABA treatments (Fig. 2a). ABA responses were highly variable depending on the gene, plant organ or duration of the treatment. Some were highly suppressed or selectively responsive such as ARABIDOPSIS TOXICOS IN LEVADURAS 8 (ATL8), XB3 ORTHOLOG 4 IN ARABIDOPSIS (XBAT34) and ARABIDOPSIS TOXICOS IN LEVADURAS 42 (ATL42), while ABA INSENSITIVE RING PROTEIN 2 (AIRP2) was up-regulated in most treatments. Most of these RING-type ubiquitin E3 ligase genes showed up-regulation after 3-h ABA treatment in seedlings, adult plants or guard cells (Fig. 2a). The gene expression data for the same 15 genes was analysed for GA responses during seed germination but no differential expression patterns compared to Col-0 were observed (Fig. S3).

\section{Validation of RING-type ubiquitin E3 ligases by RT-qPCR}

To validate the expression data extracted from Genevestigator, 9 of the 15 RING-type ubiquitin E3 ligase genes were selected and analysed by RT-qPCR for their expression profiles (Fig. 2b). As seen in the Genevestigator data, the gene expression of AT2G77770, ATL42, AIP2, AIRP2, RING-H2 FINGER AlB (RHAlB), WAV3 HOMOLOG 1 (WAVHI) and RING AND DOMAIN OF UNKNOWN FUNCTION 1117 1 (RDUF1) was up-regulated, while ARABIDOPSIS TOXICOS EN LEVADURAS 31 (ATL31) and XBAT34 were down regulated by ABA (Fig. 2a, b). AIRP2, RDUF and WAVH1 showed an early ABA response, being upregulated at $1.5 \mathrm{~h}$ of treatment. In turn, AT1G77770, ATL42, AIP2, WAWH1 and $R H A 1 B$ expression peaked moderately at $3 \mathrm{~h}$ of ABA treatment. Conversely, XBAT34 was downregulated after $3 \mathrm{~h}$ of ABA treatment. Interestingly, $0 \mu \mathrm{M}$ ABA treatment induced a strong downregulation of AIRP2. The highest differential expression in this analysis was observed in AIRP2, WAVH1 and XBAT34. These results confirmed these RINGtype ubiquitin E3 ligase genes as ABA responsive genes.

\section{Progressive drought responses of aip2-1 and wavh1-3}

To test if ABA responses were time point specific, aip21 , as ABA responding control with no drought response and wavhl-3 with similar, but stronger ABA response gene expression pattern to aip 2-1 (Fig. 2b) were subjected to progressive drought analysis. We measured the projected rosette area (PRA), maximum quantum yield of photosystem II (QY max), effective quantum yield of photosystem II ( $\phi$ PSII), non-photochemical quenching (NPQ), ratio of fluorescence decay (Rfd) and rosette temperature (Supplemental Fig. S2). No differences were observed between mutant lines and Col-0, indicating that the role of these two RING-type ubiquitin E3 ligases is linked to germination and not drought tolerance.

\section{High throughput platform for cotyledon emergency using chlorophyll fluorescence imaging}

To confirm the observed ABA responses at gene expression level, we analysed germination of the 18 T-DNA insertion lines corresponding to 15 RING-type ubiquitin E3 ligase genes at different concentrations of ABA. In this assay development, we used cotyledon emergence as a proxy for seed germination. For the first assay we tested four ABA concentrations: 0, 250, 500 and $1000 \mathrm{nM}$. The highest ABA concentration inhibited germination, while in $500 \mathrm{nM} \mathrm{ABA}$ concentration only few seedlings germinated after day 6 (data not shown). Therefore, 500 and $1000 \mathrm{nM} \mathrm{ABA}$ were omitted from the analysis and later assays. Thirty seeds per seedstock were sown in round plates in a 5 times 6 array and randomized on trays with nine plates (Fig. 1a, left and middle panels). 


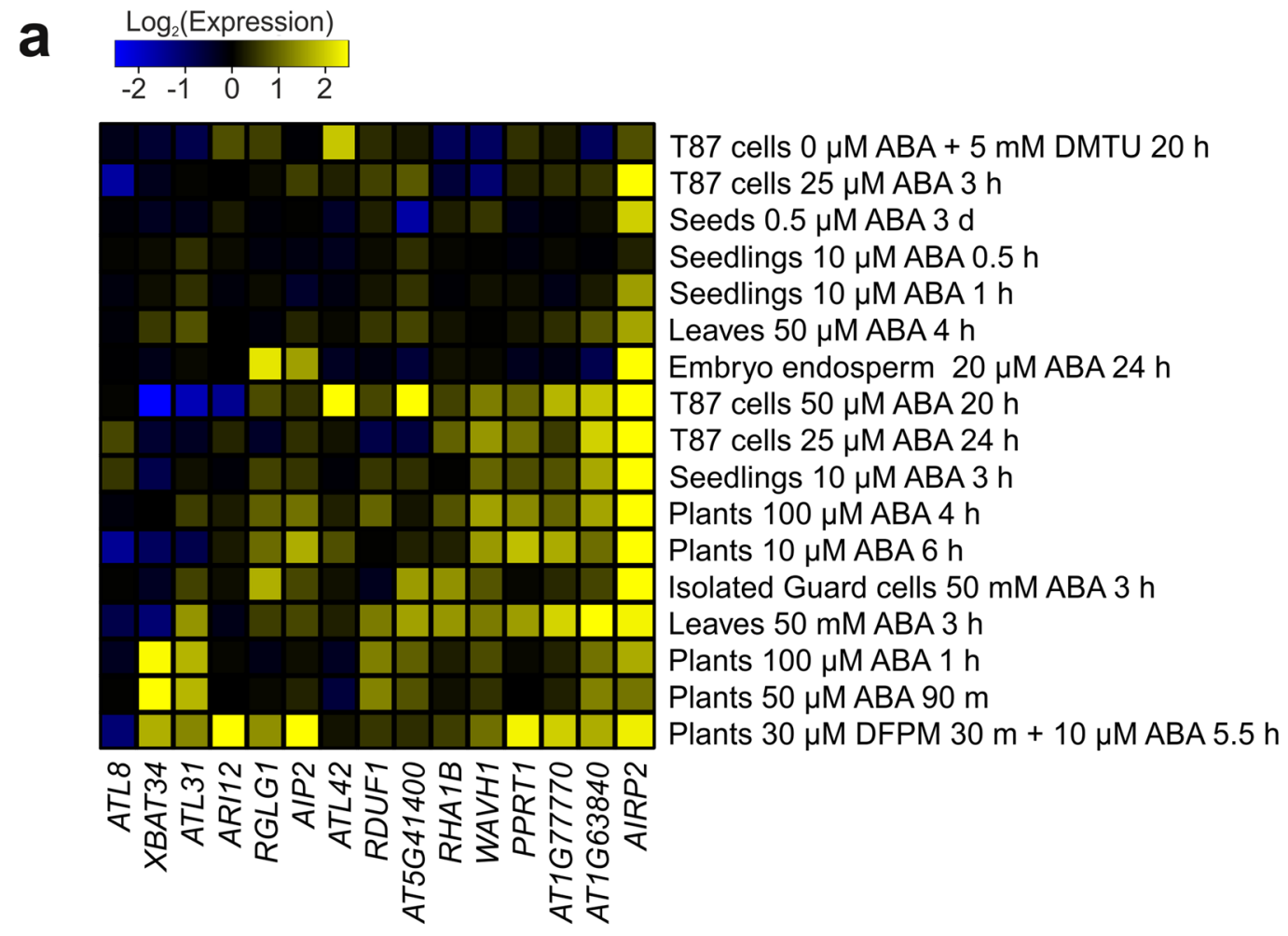

b

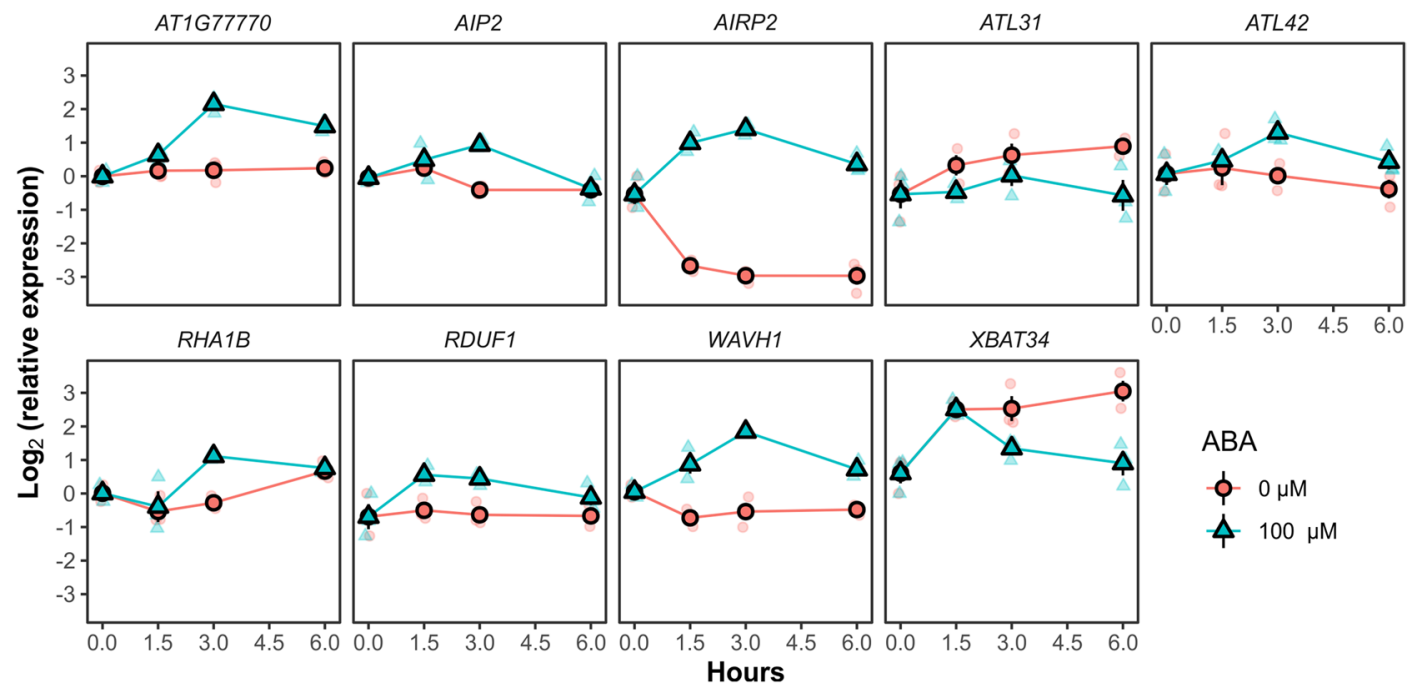

Fig. 2 Bioinformatic analysis of RING-type ubiquitin E3 ligase gene expression in ABA treatments. a Heat map of gene expression patterns of 15 RING-type ubiquitin E3 ligase genes in ABA treatments in a set of microarray experiments from Genevestigator database. Expression data with more than fourfold of change $\left(\mathrm{LOG}_{2}>2\right)$ where

To automate the detection of germination, we used $\mathrm{F}_{\mathrm{v}} /$ $\mathrm{F}_{\mathrm{m}}$ chlorophyll fluorescence imaging. Using this technique, the plates were imaged daily for 7 days after stratification. To automate the data extraction from $\mathrm{F}_{\mathrm{m}}$ images, all postprocessing steps were registered using FIJI macro recorder and then applied to a folder containing all images generated retrieved and sorted. Yellow and blue colours representing up- and down-regulation of fold-change between 2 and -2 , respectively. b Relative expression of 9 ubiquitin E3 ligase genes in 2-week old in vitro Col-0 seedlings treated with 0 and $100 \mu \mathrm{M}$ ABA for $0,1.5,3$ or $6 \mathrm{~h}$ The fold-up was calculated to $0 \mathrm{~h}(\mathrm{n}=3$, error bars $=\mathrm{SE})$

during the experiment. The first step in the macro was to import raw $\mathrm{F}_{\mathrm{m}}$ fimg images generated by PSI FluorCam system and convert them into TIFF format. Afterwards, pixels belonging only to seedlings were selected and background pixels were filtered out based on their $F_{m}$ values (Fig. 1b). After pixel thresholding, the images were converted into 
binary images and a selection rectangle was drawn around each plate and FIJI plugin Analyse Particles was run. This plugin recognizes and counts the pixel areas of seedlings within the drawn rectangle. The counts were saved in comma separated value (CSV) files with the image name that served as an identification code for posterior linking of the seedlings with their genotype and measuring time. In our assays, this process lasted about $15 \mathrm{~s}$, allowing rapid and efficient analysis of images.

\section{Manual validation of the automated system}

To evaluate the performance of the automated system, the percentage of germination was measured automatically as described above, and manually using a stereoscopic microscope. In total, 24 plates $0 \mathrm{nM}$ and 24 plates with $250 \mathrm{nM}$ ABA were analysed for 7 days, giving 336 germination measurements. In the manual estimation, seeds with cotyledons completely separated from the seed coat were considered as germinated (Boyes et al. 2001). In the automated percentage of germination measurement was estimated using the same parameters described above (seedlings with pixel value in the $\mathrm{F}_{\mathrm{m}}$ images above 180). In addition, we tested the effect of different pixel thresholding values on the automated image processing. The pixel threshold of 110 generated binary images which were biased towards manual counting, and a threshold of 250 biased the analysis towards automatic counting (data not shown). Thus, a pixel threshold of 180 generated the most similar automated percentage of germination to manual estimation (Fig. 3b). A linear model was fitted to a plot of manual counting versus automatic counting showing $\mathrm{R}^{2}$ value of 0.9643 and the slope different than zero with high significance ( $p$ value $<0.0001$ ). This analysis indicates that the automated percentage of germination measurement was highly representative of the manual estimation (Fig. 3c).

\section{Hormone germination assays of RING E3 ligases}

ABA treatment delayed germination, and therefore cotyledon emergence, in a concentration dependent manner (Fig. 3a). At day 1 after stratification no seedlings were detected in any of the treatments. On $0 \mathrm{nM}$ ABA plates the first seedlings started to show green cotyledons after 2 days in all lines (Fig. 3a). For Col-0, applying $250 \mathrm{nM}$ of ABA delayed the first cotyledon emergence to day 3 . ABA concentration of $500 \mathrm{nM}$ was enough to suppress the cotyledon emergence in all lines, only few seedlings emerged after 6 days poststratification. Taken together, the best separation between Col-0 and mutant lines was observed on $250 \mathrm{nM} \mathrm{ABA.} \mathrm{Of}$ the 18 lines, five lines showed sensitive (aip2-1, at5g414001, atl8-1, rhalb-1 and wavhl-3) and one line showed insensitive (atl42-2) responses to ABA on germination rate in comparison to Col-0 (Fig. 3a, Supplemental Fig. S1 and Supplemental Table S2). Lines wavhl-3 and atl42-2 showed strong differences on $250 \mathrm{nM} \mathrm{ABA}$, having, respectively, less or more germinated seedlings than Col-0 (Fig. 3a).

For each line the maximum percentage of germination on day 7 was calculated and mutant lines aip2-1, at5g41400-1, atl $8-1$, rhalb-1 and wavhl-3 showed below $25 \%$ of maximum percentage of germination ( $p$-value $<0.05$, Fig. $3 \mathrm{~b}$ and Supplemental Table S3).

Despite the strong resistance to ABA treatment of atl422 mutant on days 4-6, it was not different than Col-0 for maximum percentage of germination at day 7 (Fig. 3b and Supplemental Table S3). However, the trend shows still a higher average of percentage of germination for atl42-2.

\section{Discussion}

\section{Gene expression and germination screens revealed early $A B A$ response RING-type ubiquitin $E 3$ ligases}

Plants such as Arabidopsis have evolved hundreds of RINGtype ubiquitin E3 ligases to regulate their development, growth and stress response (Stone et al. 2005; Sadanandom et al. 2012; Pavicic et al. 2017; Jiménez-López et al. 2018). In our study, ABA was selected as an early developmental regulator to screen a mutant collection to identify possible new ABA response RING-type ubiquitin E3 ligases. Gene expression patterns of the selection of 36 RING-type ubiquitin E3 ligases were screened on ABA treatment in the Genevestigator transcriptome database. Altogether 15 out of the 36 genes were up or down regulated by the variety of ABA treatments (Fig. 2a). Among the 15 ABA response candidates, several known ABA response ubiquitin E3 ligases could be identified, such as AIP2, AIRP2, PROTEIN WITH THE RING DOMAIN AND TMEMB_185A 1 (PPRT1) and RDUF1 (Zhang et al. 2005; Cho et al. 2011; Kim et al. 2012; Pei et al. 2019).

Our working hypothesis was that mutants of ubiquitin E3 ligases, which were up-regulated upon $\mathrm{ABA}$ treatment, would be more resistant to ABA than Col-0. This implies that the ubiquitin E3 ligase substrate would be a repressor of the given ABA response pathway (Fig. 4). If the substrate would be an activator, then the respective ubiquitin E3 ligase mutant would show ABA hypersensitivity. To test the working hypothesis the ABA response RING-type ubiquitin E3 ligases were subjected to germination screen on ABA concentration gradient. As a control line aip2-1 behaved susceptible to ABA treatment in accordance to Zhang et al. (2005), validating the set up (Fig. 3a, b). Five ABA response RING-type ubiquitin E3 ligases were identified as responding differentially compared to Col-0 wild type: at $5 \mathrm{~g} 41400$ 1, atl42-2, atl8-1, rhalb-1 and wavh1-3 (Fig. 3a, b). With 
a

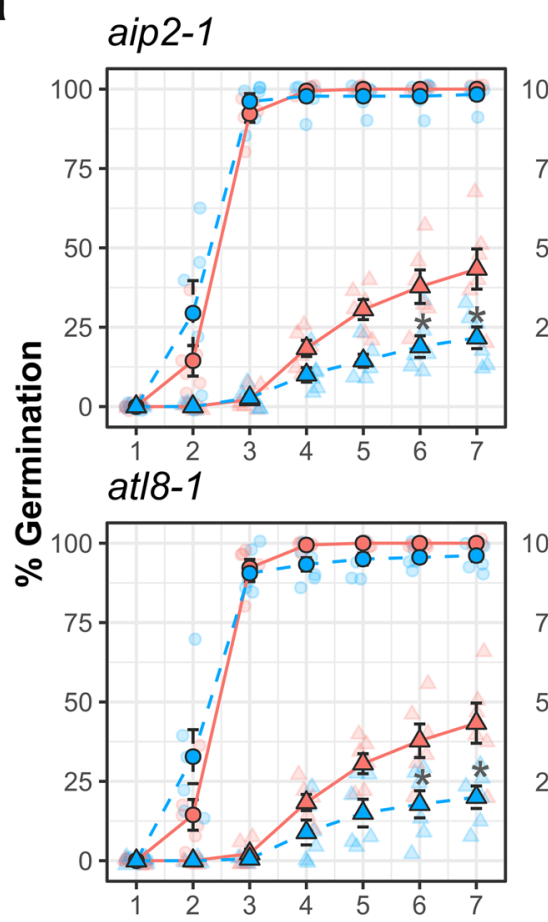

Col-0 $0 \mathrm{nM}$

$\mathrm{O}$

b

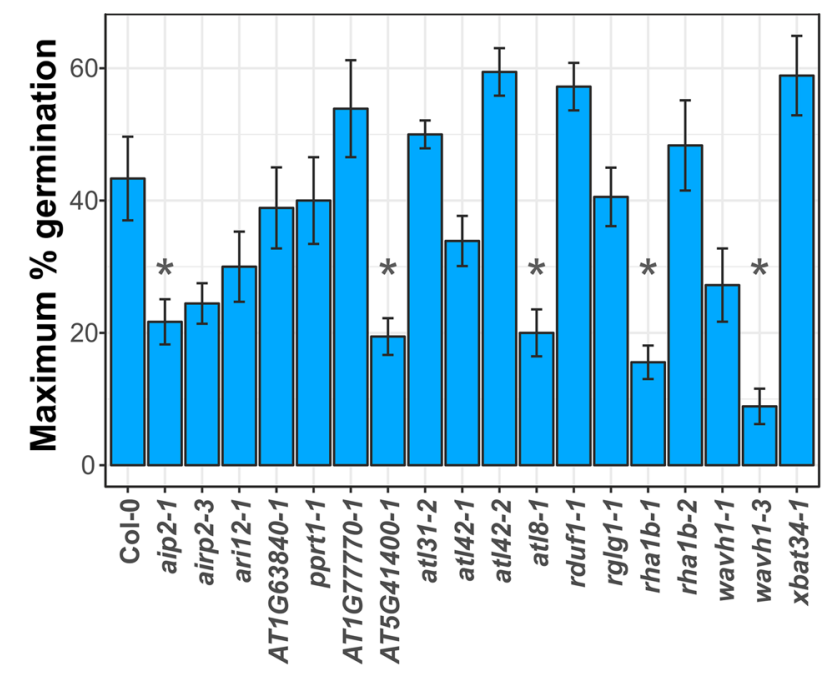

at5g41400-1

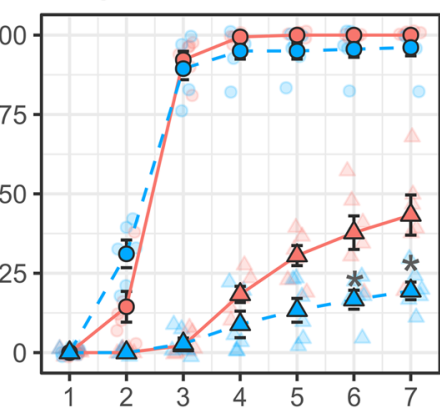
rha $1 b-1$

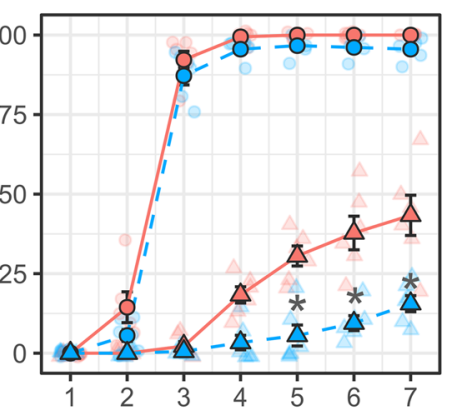

Days after stratification at/42-2

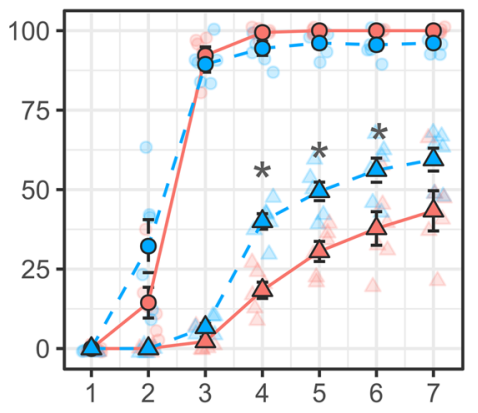

wavh1-3

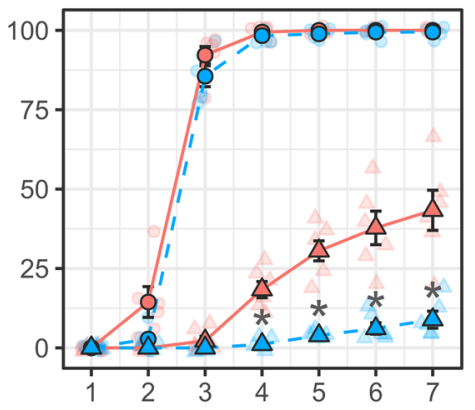

Col-0 $250 \mathrm{nM}$ mutant $0 \mathrm{nM}$ mutant $250 \mathrm{nM}$

\section{C}

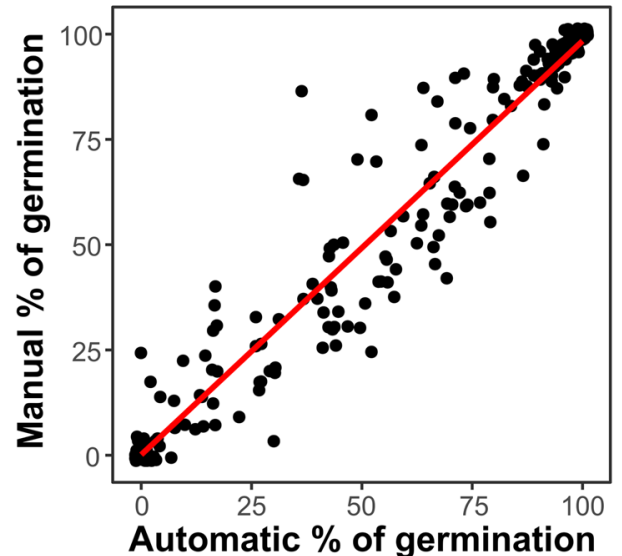

Automatic \% of germination

Fig. 3 Percentage of germination on different ABA concentrations. a Cumulative percentage of germination for six of the 18 knockout lines in media supplemented with 0 or $250 \mu \mathrm{M}$ ABA. Results represent the mean of six individual measurements of two experimental repetition. Faded point in the background represent individual measurements with a soft jittering to prevent point overlapping. b Maximum percentage of germination in medium supplemented with
$250 \mu \mathrm{M}$ ABA. c Correlation of automated and manual percentage of germination estimation. Asterisks show mutant lines (a and b) and days $\mathbf{b}$ where differences statistically significant $(p$-value $<0.05)$. Differences were calculated using ANOVA followed by Dunnett's post hoc test, adjusting $p$-values to the number of comparisons. Error bars: standard error of the mean 
Fig. 4 Hypotheses for ubiquitin E3 ligase function in $\mathrm{ABA}$ response pathways. a In the absence of ABA, the ABA signalling pathway is not activated in Col-0 due to active repression. $\mathbf{b}$ In the presence of ABA, the $\mathrm{ABA}$ response repressors that are ubiquitin E3 ligase substrates are ubiquitinated and targeted for degradation, resulting in release of ABA response. c Ubiquitin E3 ligases can act on $\mathrm{ABA}$ response pathway participating in degradation of activators or enhancers of ABA responses. $\mathbf{d}$ In a situation where the E3 ligase substrate is ABA response activator, the ABA supply will inactivate ubiquitin E3 ligase and prevent degradation of the activator, which leads to hypersensitivity to $\mathrm{ABA}$ a

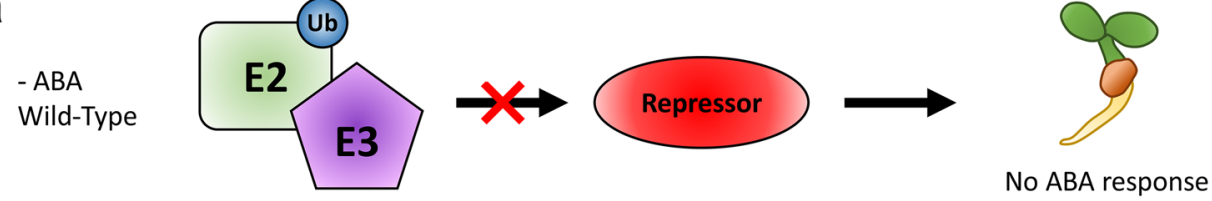

b

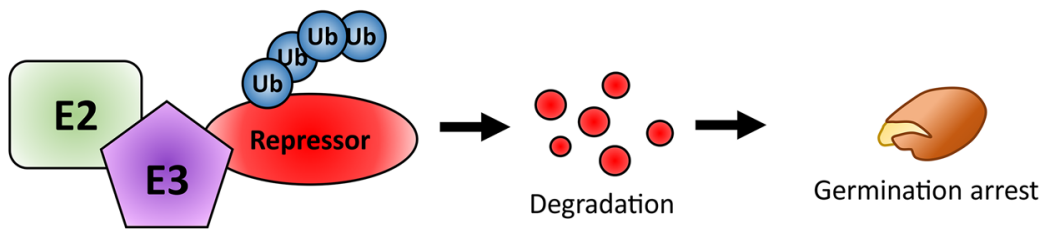

C
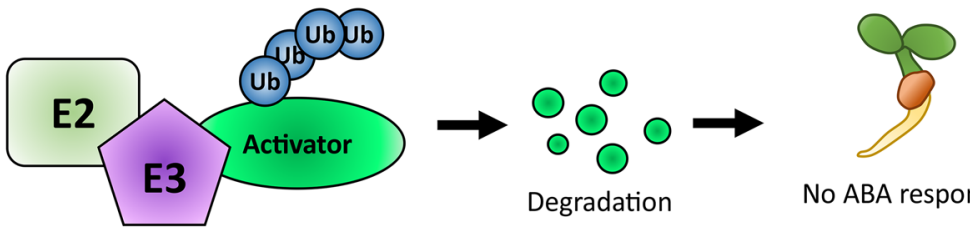
Wild-Type

$+A B A$ Wild-Type

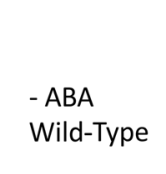

d

+ ABA Wild-Type
No $A B A$ response

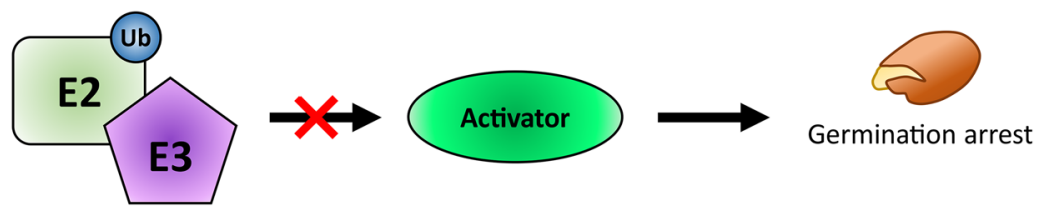

$250 \mathrm{nM}$ ABA atl42-2 line was able to germinate at $60 \%$ rate, while Col-0 was below $50 \%$ (Fig. 3a). In contrast, the other four mutant lines showed lower germination levels than Col-0 (Fig. 3). These results suggest that our screen was able to capture both types of responses proposed in our working hypothesis. In the RT-qPCR analysis AIP2, ATL42, RHA1B and $W A V H 1$ showed early ABA responses, being upregulated after 1.5 and peaking at $3 \mathrm{~h}$ after treatment. ATL42 belongs to the RING H2-type ARABIDOPSIS TOXICOS EN LEVADURAS (ATL) family and it has not been characterized. However, eFP Browser data suggests that it is a root specific gene induced by heat and cold (Schmid et al. 2005; Kilian et al. 2007; Klepikova et al. 2016). A mutant line of another member of the ATL family, ATL43, showed resistant phenotype to ABA treatments in germination (Serrano et al. 2006). ATL42 and ATL43 share high sequence similarity and same germination phenotype in ABA, suggesting that they could share similar functions (data not shown). Double knockout lines atl42/atl43 would help to elucidate their relationship during seed germination. ATL8, was described previously by Serrano et al. (2006), no homozygous mutant could be identified, and it was suggested that ATL8 could be an essential gene for Arabidopsis development. In this study, homozygous atl8-1 mutant was isolated and RT-qPCR results suggest that it is a full knockout mutant, with ABA susceptible phenotype (Fig. 3a). RHA1B phenotype has not been characterized previously and expression analysis shows that it is highly expressed in dry seeds (Schmid et al. 2005; Klepikova et al. 2016). Double mutants of RING-H2
FINGER A2A (RHA2A) and RHA2B, two closely related genes to $R H A 1 B$, are resistant to ABA treatments in cotyledon greening phenotype (Li et al. 2011). The mutant rhalb-1 showed susceptibility to ABA treatment suggesting that it could play opposite role to RHA2A and RHA2B (Fig. 3a, b). The gene $A T 5 G 41400$ is a RING H2-type gene with a very similar amino acidic sequence to RHA1B (data not shown). Expression data shows that AT5G41400 is induced by salt, drought and heat, suggesting that it could be a stress related gene (Kilian et al. 2007). WAVH1 gene also contains a RING H2-type domain and has been associated with the very first leaf primordia in young seedlings, but its association with germination has not been stablished previously (Sakai et al. 2012). The expression patterns of these genes are variable across Arabidopsis development. However, as all these genes contain a RING H2-type domain, our results suggest that this type of proteins could be hormone and stress related.

\section{UPS involvement in ABA signalling}

Germinating at proper time and in optimal conditions is critical for offspring survival and preventing pre-harvest sprouting. Defects in primary dormancy will result in pre-harvest sprouting which will lead to the loss of grain weight and quality of kernels in cereals such as wheat, rice and maize (Fang and Chu 2008). ABA has pleiotropic effects on plant development and growth, such as inhibition of seed germination and seedling growth. Additionally, ABA is a stress related hormone, and the 
ABA signalling pathways are overlapping with drought, cold and immune response (Fujita et al. 2006; Cao et al. 2011), all of which are enormous challenge to crops growing in the field (Gray et al. 1999; Pauwels et al. 2010). There is accumulating evidence that the UPS signalling and individual RING-type ubiquitin E3 ligases, such as AIP2, KEEP ON GOING (KEG), AIRP2, RHA2A and RHA2B, are involved in ABA signalling (Zhang et al. 2005; Stone et al. 2006; Lyzenga and Stone 2011; Li et al. 2011; Cho et al. 2011; Yu et al. 2016). From these known ABA related ubiquitin E3 ligases we included AIP2 in the analysis as control of ABA response. On the germination screen atl42-2 mutant seeds were resistant to ABA reaching about $60 \%$ germination, while at $5941400-1$, at $18-1$, rhalb-1 and wavhl-3 were susceptible with germination below 25\%, in ABA treatment. Therefore, we speculate that in Col-0 plant, ATL42 would target repressors, and while the other four RING H2-type proteins could target activators of ABA response for proteosomal degradation. Thus, the mechanism of action of $A B A$ regulation in Col-0 would be that without supply of ABA there is no ABA response ubiquitin E3 ligase induction. Therefore, substrates (here, ABA response repressors) remain active and repress ABA responses (Fig. 4a). In the other hand, activators of ABA pathway would be constantly degraded to prevent strong responses of this hormone (Fig. 4c). In the presence of ABA, ATL42 will target repressor substrates for degradation, leading to normal $\mathrm{ABA}$ response (Fig. 4b). In this same scenario, ABA pathway activators would be induced together with their respective E3 ubiquitin E3 ligases (such as WAVH1), allowing the right amount of activators to accumulate, and thus lead to a controlled ABA response (Fig. 4d).

The ATL42 knock out mutant where is unable to target repressor substrates for degradation, and therefore they are stabilized, and ABA responses are not activated despite ABA supply. In ubiquitin E3 ligase mutants like wavh1-3, which targets $\mathrm{ABA}$ response activators, the opposite outcome is expected. Because the ubiquitin E3 ligase is not induced upon ABA supply, ABA response activators accumulate and remain active to promote strong ABA response. Therefore, such mutant is hypersensitive to ABA like shown for aip2-1 and wavh1-2. In the ABA germination screen, aip2-1 and wavh1-2 were ABA hypersensitive. Indeed, a known AIP2 target protein $\mathrm{ABI} 3$ promotes $\mathrm{ABA}$ signalling and represses germination of seeds (Zhang et al. 2005). Substrates for WAVH1 E3 ligase are not known. We tested the mutant wavh1-3 and aip 2-1 for progressive drought assays with not significant differences from the control plants. These results suggest that for those two genes their ABA responses are specific to germination.
Automated HTP germination assay to facilitate gene function discoveries

Seed quality is an important agronomic trait that ensures uniform germination rate and seedling vigour (Rajjou et al. 2012). Recently, several approaches have been developed to automate or facilitate the seed quality assessment or analysis of the germination process (Dumont et al. 2015; Jahnke et al. 2016; Ligterink and Hilhorst 2017; Zhang et al. 2018). In these applications, facilities with high precision imaging tools provide means for analysis of seed size, volume, colour and internal quality. Eventually automation can be utilized sorting the individual seeds based on the assigned quality control (Jahnke et al. 2016). Automation can also be extended to delivery of the imaging targets to camera (this study), or camera to the imaging targets. In combination with different levels of automation high throughput plant phenotyping facilities would allow assessing the germination processes in time course fashion. Here, to allow fast screening of the tens of mutant lines for germination on several concentrations of ABA, a high throughput imaging-based assay was established. To this end, Arabidopsis seeds germinating on the petri dishes containing different concentrations of ABA hormone were imaged by chlorophyll fluorescence sensor. The cotyledon emergence was used as an indicator of germination to allow detecting the tiny Arabidopsis seedlings by the chlorophyll fluorescence signal (Fig. 1). For larger seeds, such as wheat and legumes, RGB imaging and colour-based detection can be utilized instead of chlorophyll fluorescence (Zhang et al. 2018). The image processing workflow allowed detecting the very first fluorescence signals that the emerging cotyledons emitted, and these signals were used for scoring the germination rate of the mutant lines. Automation of this process allowed screening of hundreds of pictures in few minutes. This data extraction together with $\mathrm{R}$ script pipeline allowed to go from raw images to exploratory plots in less than $30 \mathrm{~min}$. Thus, establishing high throughput germination screens in the automated imaging-based phenotyping facilities will facilitate analysis of seed quality traits such as germination rate, dormancy and seed longevity but also bioassays with hormones and compound libraries.

UPS mediated protein degradation has been found to participate in numerous of signalling pathways in plants, including phytohormone mediated plant growth and development (Gray et al. 1999; Pauwels et al. 2010). The automated high throughput germination screen allowed us to discover two novel ubiquitin E3 ligases associated with ABA signalling pathways. The two novel ABA signalling RING-type ubiquitin E3 ligases identified here are currently being functionally characterized to push forward current understanding of ubiquitin E3 ligases as a molecular switch from growth and development to stress responses. Future work will aim 
at identification of their molecular networks, including the substrates.

Acknowledgements Open access funding provided by University of Helsinki including Helsinki University Central Hospital.

Author contributions $\mathrm{KH}, \mathrm{MP}$ and $\mathrm{FW}$ designed the experiments, MP and FW performed the bioinformatic analysis, FW, KM and $\mathrm{KH}$ characterized the transgenic lines, KH designed the phenotyping platform, MP and FW performed the HTP germination assays, MP performed the image processing and statistical analysis of data, FW and MP performed the manual germination assays, KM performed the drought assay, $\mathrm{KH}$ wrote the manuscript together with KM, FW and MP. All four authors agreed about the submission.

\section{Compliance with ethical standards}

Conflict of interest Here, for our original research titled "High throughput in vitro seed germination screen identified new ABA responsive RING-type ubiquitin E3 ligases in Arabidopsis thaliana" we declare there is no known conflict of interest associated with this publication.

Open Access This article is distributed under the terms of the Creative Commons Attribution 4.0 International License (http://creativeco mmons.org/licenses/by/4.0/), which permits unrestricted use, distribution, and reproduction in any medium, provided you give appropriate credit to the original author(s) and the source, provide a link to the Creative Commons license, and indicate if changes were made.

\section{References}

Abid G, M'hamdi M, Mingeot D et al (2017) Effect of drought stress on chlorophyll fluorescence, antioxidant enzyme activities and gene expression patterns in faba bean (Vicia faba L.). Early Interv Psychiatry. https://doi.org/10.1080/03650340.2016.1224857

Adler G, Konrad Z, Zamir L et al (2017) The Arabidopsis paralogs, PUB46 and PUB48, encoding U-box E3 ubiquitin ligases, are essential for plant response to drought stress. BMC Plant Biol. https://doi.org/10.1186/s12870-016-0963-5

Boyes DC, Zayed AM, Ascenzi R et al (2001) Growth stage-based phenotypic analysis of Arabidopsis: a model for high throughput functional genomics in plants. Plant Cell 13:1499-1510. https:// doi.org/10.1105/tpc.010011

Cao FY, Yoshioka K, Desveaux D (2011) The roles of ABA in plantpathogen interactions. J Plant Res 124:489-499. https://doi. org/10.1007/s10265-011-0409-y

Cho SK, Ryu MY, Seo DH et al (2011) The Arabidopsis RING E3 ubiquitin ligase AtAIRP2 plays combinatory roles with AtAIRP1 in abscisic acid-mediated drought stress responses. Plant Physiol 157:2240-2257. https://doi.org/10.1104/pp.111.185595

Czechowski T, Stitt M, Altmann T et al (2005) Genome-wide identification and testing of superior reference genes for transcript normalization in Arabidopsis. Plant Physiol 139:5-17. https://doi. org/10.1104/pp.105.063743

Dumont J, Hirvonen T, Heikkinen V et al (2015) Thermal and hyperspectral imaging for Norway spruce (Picea abies) seeds screening. Comput Electron Agric 116:118-124. https://doi.org/10.1016/j. compag.2015.06.010

Fang J, Chu C (2008) Abscisic acid and the pre-harvest sprouting in cereals. Plant Signal Behav 3:1046-1048

Fujita M, Fujita Y, Noutoshi Y et al (2006) Crosstalk between abiotic and biotic stress responses: a current view from the points of convergence in the stress signaling networks. Curr Opin Plant Biol 9:436-442

Goda H, Sasaki E, Akiyama K et al (2008) The AtGenExpress hormone and chemical treatment data set: experimental design, data evaluation, model data analysis and data access. Plant J 55:526-542. https://doi.org/10.1111/j.1365-313X.2008.03510.x

Gou M, Huang Q, Qian W et al (2017) Sumoylation E3 ligase SIZ1 modulates plant immunity partly through the immune receptor gene SNC1 in Arabidopsis. Mol Plant-Microbe Interact 30:334 342. https://doi.org/10.1094/MPMI-02-17-0041-R

Gray WM, Del Pozo JC, Walker L et al (1999) Identification of an SCF ubiquitin-ligase complex required for auxin response in Arabidopsis thaliana. Genes Dev 13:1678-1691. https://doi.org/10.1101/ gad.13.13.1678

Hothorn T, Bretz F, Westfall P (2008) Simultaneous inference in general parametric models. Biom J 50:346-363. https://doi. org/10.1002/bimj.200810425

Hruz T, Laule O, Szabo G et al (2008) Genevestigator v3: a reference expression database for the meta-analysis of transcriptomes. Adv Bioinform 2008:1-5. https://doi.org/10.1155/2008/420747

Jahnke S, Roussel J, Hombach T et al (2016) PhenoSeeder-A robot system for automated handling and phenotyping of individual seeds. Plant Physiol 173:1358-1370. https://doi.org/10.1104/ pp.16.01122

Jiménez-López D, Muñóz-Belman F, González-Prieto JM et al (2018) Repertoire of plant RING E3 ubiquitin ligases revisited: new groups counting gene families and single genes. PLoS ONE 13:e0203442. https://doi.org/10.1371/journal.pone.0203442

Joosen RVL, Kodde J, Willems LAJ et al (2010) GERMINATOR: a software package for high-throughput scoring and curve fitting of Arabidopsis seed germination. Plant J 62:148-159. https://doi. org/10.1111/j.1365-313X.2009.04116.x

Kilian J, Whitehead D, Horak J et al (2007) The AtGenExpress global stress expression data set: protocols, evaluation and model data analysis of UV-B light, drought and cold stress responses. Plant J 50:347-363. https://doi.org/10.1111/j.1365-313X.2007.03052.x

Kim SJ, Ryu MY, Kim WT (2012) Suppression of Arabidopsis RING-DUF1117 E3 ubiquitin ligases, AtRDUF1 and AtRDUF2, reduces tolerance to $\mathrm{ABA}$-mediated drought stress. Biochem Biophys Res Commun 420:141-147. https://doi.org/10.1016/J. BBRC.2012.02.131

Klepikova AV, Kasianov AS, Gerasimov ES et al (2016) A high resolution map of the Arabidopsis thaliana developmental transcriptome based on RNA-seq profiling. Plant J 88:1058-1070. https://doi. org/10.1111/tpj.13312

Li H, Jiang H, Bu Q et al (2011) The Arabidopsis RING finger E3 ligase RHA2b acts additively with RHA2a in regulating abscisic acid signaling and drought response. Plant Physiol 156:550-563. https://doi.org/10.1104/pp.111.176214

Ligterink W, Hilhorst HWM (2017) High-throughput scoring of seed germination. Plant hormones. Springer, New York, pp 57-72

Lyzenga WJ, Stone SL (2011) Abiotic stress tolerance mediated by protein ubiquitination. J Exp Bot 63:599-616

Murchie EH, Lawson T et al (2013) Chlorophyll fluorescence analysis: a guide to good practice and understanding some new applications. J Exp Bot 64:3983-3998. https://doi.org/10.1093/jxb/ert208

Ni W, Xie D, Hobbie L et al (2004) Regulation of flower development in Arabidopsis by SCF complexes. Plant Physiol. https:// doi.org/10.1104/pp.103.031971

Pauwels L, Barbero GF, Geerinck J et al (2010) NINJA connects the co-repressor TOPLESS to jasmonate signalling. Nature 464:788 791. https://doi.org/10.1038/nature08854

Pavicic M, Mouhu K, Wang F et al (2017) Genomic and phenomic screens for flower related ring type ubiquitin E3 ligases in Arabidopsis. Front Plant Sci 8:416. https://doi.org/10.3389/ fpls.2017.00416 
Pei L, Peng L, Wan X et al (2019) Expression pattern and function analysis of AtPPRT1, a novel negative regulator in ABA and drought stress responses in Arabidopsis. Int J Mol Sci 20:394. https://doi.org/10.3390/ijms20020394

Rajjou L, Duval M, Gallardo K et al (2012) Seed germination and vigor. Annu Rev Plant Biol 63:507-533. https://doi.org/10.1146/ annurev-arplant-042811-105550

Rueden CT, Schindelin J, Hiner MC et al (2017) Image J2: ImageJ for the next generation of scientific image data. BMC Bioinform 18:529. https://doi.org/10.1186/s12859-017-1934-z

Sadanandom A, Bailey M, Ewan R et al (2012) The ubiquitin-proteasome system: central modifier of plant signalling. New Phytol 196:13-28. https://doi.org/10.1111/j.1469-8137.2012.04266.x

Sakai T, Mochizuki S, Haga K et al (2012) The wavy growth 3 E3 ligase family controls the gravitropic response in Arabidopsis roots. Plant J 70:303-314. https://doi.org/10.1111/j.1365313X.2011.04870.x

Schindelin J, Arganda-Carreras I, Frise E et al (2012) Fiji: an opensource platform for biological-image analysis. Nat Methods 9:676-682. https://doi.org/10.1038/nmeth.2019

Schmid M, Davison TS, Henz SR et al (2005) A gene expression map of Arabidopsis thaliana development. Nat Genet 37:501-506. https://doi.org/10.1038/ng1543

Schmittgen TD, Livak KJ (2008) Analyzing real-time PCR data by the comparative CT method. Nat Protoc 3:1101-1108. https://doi. org/10.1038/nprot.2008.73

Serrano M, Parra S, Alcaraz LD, Guzmán P (2006) The ATL gene family from Arabidopsis thaliana and Oryza sativa comprises a large number of putative ubiquitin ligases of the RING-H2 type. J Mol Evol 62:434-445

Stone SL (2014) The role of ubiquitin and the 26S proteasome in plant abiotic stress signaling. Front Plant Sci 5:135

Stone SL, Hauksdóttir H, Troy A et al (2005) Functional analysis of the RING-type ubiquitin ligase family of Arabidopsis. Plant Physiol 137:13-30. https://doi.org/10.1104/pp.104.052423

Stone SL, Williams L, Farmer LM et al (2006) KEEP ON GOING, a RING E3 ligase essential for Arabidopsis growth and development, is involved in abscisic acid signaling. Plant Cell 18:34153428. https://doi.org/10.1105/tpc.106.046532
Tan X, Calderon-Villalobos LIA, Sharon M et al (2007) Mechanism of auxin perception by the TIR 1 ubiquitin ligase. Nature 446:640 645. https://doi.org/10.1038/nature05731

Tian M, Lou L, Liu L et al (2015) The RING finger E3 ligase STRF1 is involved in membrane trafficking and modulates salt-stress response in Arabidopsis thaliana. Plant J 82:81-92. https://doi. org/10.1111/tpj.12797

Tschiersch H, Junker A, Meyer RC, Altmann T (2017) Establishment of integrated protocols for automated high throughput kinetic chlorophyll fluorescence analyses. Plant Methods. https://doi. org/10.1186/s13007-017-0204-4

Ugena L, Hýlová A, Podlešáková K et al (2018) Characterization of biostimulant mode of action using novel multi-trait high-throughput screening of Arabidopsis germination and rosette growth. Front Plant Sci 9:1327. https://doi.org/10.3389/fpls.2018.01327

Vishal B, Kumar PP (2018) Regulation of seed germination and abiotic stresses by gibberellins and abscisic acid. Front Plant Sci 9:838. https://doi.org/10.3389/FPLS.2018.00838

Whan AP, Smith AB, Cavanagh CR et al (2014) GrainScan: a low cost, fast method for grain size and colour measurements. Plant Methods 10:23

Yu F, Wu Y, Xie Q (2016) Ubiquitin-proteasome system in ABA signaling: from perception to action. Mol Plant 9:21-33. https://doi. org/10.1016/J.MOLP.2015.09.015

Zhang X, Garreton V, Chua N-H (2005) The AIP2 E3 ligase acts as a novel negative regulator of ABA signaling by promoting $\mathrm{ABI} 3$ degradation. Genes Dev 19:1532-1543. https://doi.org/10.1101/ gad.1318705

Zhang C, Si Y, Lamkey J et al (2018) High-throughput phenotyping of seed/seedling evaluation using digital image analysis. Agronomy 8:63. https://doi.org/10.3390/agronomy 8050063

Publisher's Note Springer Nature remains neutral with regard to jurisdictional claims in published maps and institutional affiliations. 